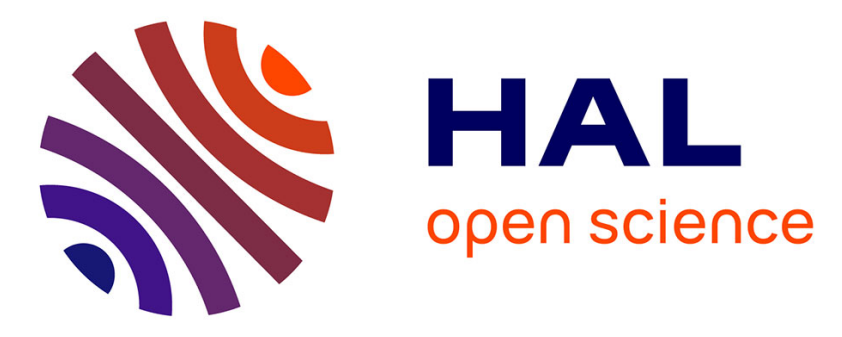

\title{
Neoclassical physics in full distribution function gyrokinetics
}

Guilhem Dif-Pradalier, P H Diamond, Virginie Grandgirard, Yanick Sarazin, J Abiteboul, Xavier Garbet, Philippe Ghendrih, Guillaume Latu, A. Strugarek, S Ku, et al.

\section{To cite this version:}

Guilhem Dif-Pradalier, P H Diamond, Virginie Grandgirard, Yanick Sarazin, J Abiteboul, et al.. Neoclassical physics in full distribution function gyrokinetics. Physics of Plasmas, 2011, 18, pp.62309. $10.1063 / 1.3592652$. cea-01468378

\section{HAL Id: cea-01468378 https://hal-cea.archives-ouvertes.fr/cea-01468378}

Submitted on 16 Feb 2017

HAL is a multi-disciplinary open access archive for the deposit and dissemination of scientific research documents, whether they are published or not. The documents may come from teaching and research institutions in France or abroad, or from public or private research centers.
L'archive ouverte pluridisciplinaire HAL, est destinée au dépôt et à la diffusion de documents scientifiques de niveau recherche, publiés ou non, émanant des établissements d'enseignement et de recherche français ou étrangers, des laboratoires publics ou privés. 


\section{Neoclassical physics in full distribution function gyrokinetics}

G. Dif-Pradalier', P. H. Diamond', V. Grandgirard, Y. Sarazin, J. Abiteboul, X. Garbet, Ph. Ghendrih, G. Latu, A. Strugarek, S. Ku, and C. S. Chang'

Citation: Phys. Plasmas 18, 062309 (2011); doi: 10.1063/1.3592652

View online: http://dx.doi.org/10.1063/1.3592652

View Table of Contents: http://aip.scitation.org/toc/php/18/6

Published by the American Institute of Physics

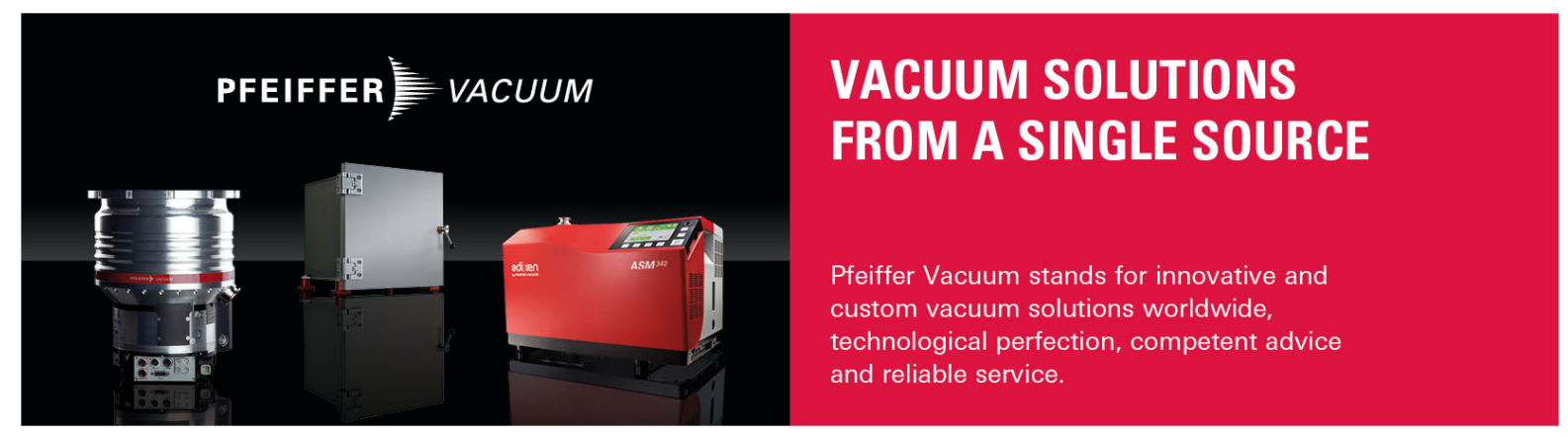




\title{
Neoclassical physics in full distribution function gyrokinetics
}

\author{
G. Dif-Pradalier, ${ }^{1, a)}$ P. H. Diamond, ${ }^{1, b)}$ V. Grandgirard, ${ }^{2}$ Y. Sarazin, ${ }^{2}$ J. Abiteboul, ${ }^{2}$ \\ X. Garbet, ${ }^{2}$ Ph. Ghendrih, ${ }^{2}$ G. Latu, ${ }^{2}$ A. Strugarek, ${ }^{2}$ S. Ku, ${ }^{3}$ and C. S. Chang ${ }^{3, c)}$ \\ ${ }^{1}$ Center for Astrophysics and Space Sciences, UCSD, La Jolla, California 92093, USA and Center \\ for Momentum Transport and Flow Organisation, UCSD, La Jolla, California 92093, USA \\ ${ }^{2}$ CEA, IRFM, Saint-Paul-lez-Durance, F-13108, France \\ ${ }^{3}$ Courant Institute of Mathematical Sciences, New York University, New York 10012, USA
}

(Received 3 March 2011; accepted 2 May 2011; published online 30 June 2011)

Treatment of binary Coulomb collisions when the full gyrokinetic distribution function is evolved is discussed here. A spectrum of different collision operators is presented, differing through both the physics that can be addressed and the numerics they are based on. Eulerian-like (semiLagrangian) and particle in cell (PIC) (Monte-Carlo) schemes are successfully cross-compared, and a detailed confrontation to neoclassical theory is shown. (C) 2011 American Institute of Physics. [doi:10.1063/1.3592652]

\section{INTRODUCTION}

From a thermodynamics standpoint, a tokamak plasma is best understood as an open system, exchanging heat, matter, and momentum with its environment. Therefore, given both a distribution of sources and sinks and evolution laws for the plasma internal dynamics, one of the more central questions one may ask translates as "What will an attracting fixed point for the dynamics look like?" Mounting evidences suggest that the ability to describe self-organisation at mesoscales and larger is the key to answering the latter question and certainly represents a new challenging problem for gyrokinetics.

Beyond its academic interest, this question has important practical consequences on our understanding of flow organisation, ${ }^{1}$ of the hierarchy of shears, ${ }^{2}$ and of the nature of transport itself. These facts have been emphasised both theoretically: through the description of avalanching and spreading, ${ }^{3-9}$ through the characterisation of nonlocal, nondiffusive behaviour ${ }^{1,10-12}$ and experimentally through some yet-to-be-understood experimental jigsaws: deep inconsistencies with a (fixed gradient) local and diffusive modeling have indeed been reported in perturbative (either hot or cold pulse) experiments, ${ }^{13-17}$ off-axis heating experiments, ${ }^{19,20}$ or whilst reporting Bohm-like scalings of the energy confinement time. ${ }^{18}$

An accurate description of such dynamics requires the simultaneous and self-consistent treatment of the full gyrokinetic distribution function (full- $f$ modeling), in full-torus (global) tokamak geometry and for a prescribed distribution of sources and sinks (flux-driven description). The first two allow for a self-consistent interplay of the dynamics at all scales, from the quickly evolving microscale of the turbulence to the slow macroscale evolution of neoclassical (NC)

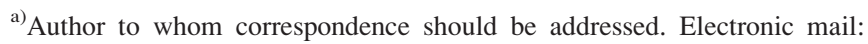
guilhem.dif-pradalier@cea.fr.

b) Present address: National Fusion Research Institute, Daejeon 305-806, South Korea.

${ }^{c}$ Also at: Department of Physics, Korea Advanced Institute of Science and Technology, Daejeon 305-701, South Korea.
}

(collisional) processes and of the background profiles, while the latter allows for a realistic description of the fuelling and sustainment processes within the plasma.

This paper focuses on a subset of this global picture and aims at discussing collisional processes within a full- $f$ gyrokinetic formalism. ${ }^{21} \mathrm{NC}$ theory ${ }^{22}$ was earlier coined to describe the effects of binary Coulomb collisions in an inhomogeneous toroidal magnetic field. Though hot plasmas are usually very weakly collisional and transport in tokamaks is rarely neoclassical-it is dominantly turbulent, an accurate accounting of collisional processes is nonetheless of major importance for modeling activities:

1. Collisions provide an ever-existing background cross-field transport mechanism, important in cases where the turbulence is quenched or strongly reduced, as happens close to marginality (for weak drives of the turbulence), in transport barrier regimes (pedestal region in $\mathrm{H}$-mode, gradient region in Internal Transport Barriers) or in stellarators.

2. The inclusion of collisional processes is indispensable to realising a statistical steady-turbulent state whilst satisfying an $\mathrm{H}$-theorem $;{ }^{21}$ on more practical grounds, the dissipation of the short-scale structures in velocity space $^{23,24}$ is also beneficial for improving numerical stability.

3. The so-called zonal and mean flows, ${ }^{25}$ well known to survive the collisionless linear Landau damping process ${ }^{26}$ are damped through collisions alone $\mathrm{e}^{27}$ in the linear regime. The fundamental importance of such sheared $\mathbf{E} \times \mathbf{B}$ flows for an accurate prediction of the level of transport has long been emphasised; neoclassical processes, through shear flow regulation, may thus participate in the saturation of the turbulence and in the plasma dynamics. ${ }^{2,28-30}$

4. An increasing number of experimental observations ${ }^{31-35}$ insists on the central role of plasma rotation (toroidal and poloidal) for the onset and control of enhanced confinement regimes and often report strong departures of poloidal rotation from its oft-expected neoclassical predictionpossibly due to the turbulence ${ }^{2,36,37}$ —either before, at, or enduring past the transition to an enhanced confinement state. 
5. Collisions at last contribute to define the long-time statistical equilibrium whilst providing, as well-known, an effective return force "towards Maxwellianity".

This latter point has important practical implications for a newer generation of gyrokinetic codes which resolve the full distribution function, ${ }^{38-44}$ amongst which the Gysela ${ }^{38}$ and $\mathrm{XGC} 1^{45}$ codes featured here. Self-consistent evolution of the distribution function is key to these models. The longtime equilibrium distribution function for the collisional gyrokinetic system is non-trivial, is not tied to an initial-often Maxwellian - choice, and realises in practice a compromise between a Maxwellian and an arbitrary function of the motion invariants. ${ }^{46-48}$

Two appreciably different choices of collision operators allowing to carefully compute neoclassical processes for the full distribution function in global magnetic geometry and with comparable precision are now presented; one best suited for Eulerian or semi-Lagrangian numerical schemes (implemented in Gysela), the other one for Monte-Carlo processes and particle-in-cell (PIC) schemes (implemented in XGC1). This study also provides a successful cross-validation of both codes, especially interesting before moving to the more physically relevant open lands of turbulence and neoclassical interplay ${ }^{2,49}$ for which a comprehensive theoretical background is often found to be lacking.

The simulations reported here are thus performed below the ion temperature gradient (ITG) linear instability threshold, with no turbulence. The accurate computation of Coulomb collisional processes in a hot plasma is notoriously complex and has not been addressed in full- $f$ modeling until very recently. ${ }^{2,44,50,51}$ As well known, the concept alone of a collision in such a medium is rather subtle, and since collisions between particles depend on their relative velocities, the overall collisional result is an integrated effect of nonlocal interactions between particles of all velocities.

An efficient computation in the Eulerian-like structure of the Grsela code (Sec. II A) will greatly differ from a similarly efficient computation in the PIC structure of the XGC1 code (Sec. II B). In each case, the collision operator is precisely tailored to the specific strengths and shortcomings of each approach in terms of resolution methods, parallelisation choices, etc. Model operators ${ }^{21}$ which allow to recover the main neoclassical results whilst preserving some interesting simplicity are thus especially attractive. The physical features that can be modeled with each collision operator are thoroughly discussed in Sec. III. Quantitative confrontation to conventional neoclassical predictions is presented throughout Sec. IV. We especially show accurate recovery of the predicted neoclassical transport, the collisional zonal and mean flow damping, and the aspect ratio dependence of poloidal rotation, along with its reversal depending on the collisionality regime.

\section{COLLISION OPERATORS IN FULL_F GYROKINETICS}

In both approaches, the radial electric field $E_{r}$, the equilibrium poloidal $v_{\theta}$ and toroidal $v_{\varphi}$ flows, and the thermodynamic forces - through evolution of the mean temperature profile in turbulent regimes-are self-consistently evolved. The electrostatic branches-slab, toroidal, and trapped modes - of the ITG instability are modeled whilst solving the coupled system of the collisional gyrokinetic equation

$$
\begin{aligned}
& \frac{\partial \bar{f}}{\partial t}+\left(\mathbf{v}_{\mathbf{E} \times \mathbf{B}}+\mathbf{v}_{\nabla B}+\mathbf{v}_{c}\right) \cdot \nabla \bar{f}+v_{\|} \nabla_{\|} \bar{f}+\frac{\mathrm{dv}_{\|}}{\mathrm{dt}} \partial_{v_{\|}} \bar{f} \\
& =\mathcal{S}(\bar{f})+\mathcal{C}(\bar{f})
\end{aligned}
$$

and quasi-neutrality equation

$$
\begin{gathered}
\frac{e}{T_{e}}[\phi-\langle\phi\rangle]-\frac{1}{n_{e q}} \nabla_{\perp} \cdot\left[\frac{m n_{e q}}{e B^{2}} \nabla_{\perp} \phi\right] \\
=\frac{1}{n_{e q}} \iint \frac{2 \pi B_{\|}^{*}}{m} \mathrm{~d} v_{\|} \mathrm{d} \mu\left(\bar{f}-\bar{f}_{\text {init }}\right)
\end{gathered}
$$

Notations and further details on the system may be found in Appendix. The electronic response is here assumed to be adiabatic: $\delta n_{e} / n_{e q}=e[\phi-\langle\phi\rangle] / T_{e}(r), \quad$ where $\langle\phi\rangle \equiv \iint \phi$; $d \theta d \varphi / \mathbf{B} \cdot \nabla \theta / \iint d \theta d \varphi / \mathbf{B} \cdot \nabla \theta$ accounts for the magnetic flux-surface average of the electric potential $\phi$ which is initially a perturbation. The ion Larmor radius is $\rho_{i}, m$ is the ion mass, $\bar{f}_{\text {init }}$ refers to the initial ion gyroaveraged distribution function, and $v_{\|}$is the velocity along the field lines. Note that a further simplification of the problem is here considered since the effects of geometry (elongation and triangularity) are not addressed in the current paper: the magnetic surfaces are indeed nested, circular, and concentric.

Acknowledging that a tokamak is indeed an open system which should be described on a statistical basis, sustainment of a steady-turbulence state only occurs on the basis of compensating for the decay of the plasma free energy (decay of the mean pressure profile) with an externally imposed heat flux coming from the environment, $\mathcal{S}(\bar{f})$ in Eq. (1). This "fluxdrive" capability ${ }^{51-55}$ allows for long-lived turbulent simulations, from a collision time up to the energy confinement time, with a statistically constant drive of the turbulence whilst selfconsistently describing the dynamics of the mean profiles. The assumption of a "fixed background gradient" is thus relaxed. This capability will not be used in the present paper; this source term is thus set to zero for neoclassical simulations, and the temperature is fixed at the boundaries. It becomes central in simulations of turbulence and is further discussed in a forthcoming paper. ${ }^{49}$ The last term in Eq. (1), the collision operator $\mathcal{C}(\bar{f})$, is now discussed for both approaches.

\section{A. For Eulerian or semi-Lagrangian schemes}

A generic energy and momentum-conserving collision scheme has recently been derived ${ }^{21}$ and implemented ${ }^{56}$ in GYSELA. It is especially well suited for Eulerian or semi-Larangian numerical schemes in which the full distribution function $f$ is evolved

$$
\mathcal{C}(f)=\frac{1}{B_{\|}^{\star}} \partial_{v_{\|}}\left\{B_{\|}^{\star} \mathcal{D}_{\|} f_{S M} \partial_{v_{\|}}\left(\frac{f}{f_{S M}}\right)\right\},
$$

where $f_{S M}$ is the shifted Maxwellian $f_{S M}=n /(2 \pi T / m)^{3 / 2}$ $\exp (-E / T), m$ is the ion mass, $E$ is the energy $E=m$ $\left(v_{\|}-V_{\|}\right)^{2} / 2+\mu B$, and $\mu$ is the adiabatic invariant. The 
conservation properties are ensured by constraining $\mathcal{D}_{\|}$to depend on $\mu$ only and defining the local fluid velocity $V_{\|}$and ion temperature $T$ as follows:

$$
\begin{aligned}
\mathfrak{p} V_{\|}= & \left\langle\frac{1}{B_{\|}^{\star}} \partial_{v_{\|}}\left(B_{\|}^{\star} m v_{\|} \mathcal{D}_{\|}\right)\right\rangle\left\langle v_{\|} \mathcal{D}_{\|}\right\rangle \\
& -\left\langle m v_{\|}^{2} \mathcal{D}_{\|}\right\rangle\left\langle\frac{1}{B_{\|}^{\star}} \partial_{v_{\|}}\left(B_{\|}^{\star} \mathcal{D}_{\|}\right)\right\rangle \\
\mathfrak{p} T= & \left\langle m^{2} v_{\|}^{2} \mathcal{D}_{\|}\right\rangle\left\langle\mathcal{D}_{\|}\right\rangle-\left\langle m v_{\|} \mathcal{D}_{\|}\right\rangle^{2}
\end{aligned}
$$

where $\langle\cdots\rangle=\int B_{\|}^{\star} d \mu d v_{\|} \bar{f} \cdots$ denotes the integral over velocity space and

$$
\begin{aligned}
\mathfrak{p}= & \left\langle\frac{1}{B_{\|}^{\star}} \partial_{v_{\|}}\left(B_{\|}^{\star} m v_{\|} \mathcal{D}_{\|}\right)\right\rangle\left\langle\mathcal{D}_{\|}\right\rangle \\
& -\left\langle m v_{\|} \mathcal{D}_{\|}\right\rangle\left\langle\frac{1}{B_{\|}^{\star}} \partial_{v_{\|}}\left(B_{\|}^{\star} \mathcal{D}_{\|}\right)\right\rangle
\end{aligned}
$$

The latter set of equations reduces to

$$
\begin{gathered}
V_{\|}=\frac{\left\langle\mathcal{D}_{\|} v_{\|}\right\rangle}{\left\langle\mathcal{D}_{\|}\right\rangle} \\
T=m \frac{\left\langle\mathcal{D}_{\|}\left(v_{\|}-V_{\|}\right)^{2}\right\rangle}{\left\langle\mathcal{D}_{\|}\right\rangle}
\end{gathered}
$$

in the case where $B_{\|}^{\star}=B$. Note that even though the collision operator [Eq. (3)] does depend on the energy, the advection is only performed in the parallel direction. The neoclassical equilibrium can indeed be accurately recovered, as will be shown in Sec. IV, without a transverse diffusion operator-an initially Maxwellian distribution function in the transverse $\mu$ direction closely remains Maxwellian as time evolves due to the invariance of $\mu$, i.e., without involving derivatives with respect to parameter $\mu$. This is especially useful in Eulerian or semi-Lagrangianbased codes like GySELA which are gridded on the gyrocenter parallel velocity and adiabatic invariant, as compared to more usual model operators using pitch angle and energy variables. The parallelisation in Gysela is performed in the radial, poloidal, and $\mu$ directions; ${ }^{57,58}$ since the parallelisation over velocity space is here intrinsic, this approach leads to a high numerical efficiency: each processor, or set of processors, has distinct values for $\mu$ and can thus perform the momentum and energy-conserving collisions locally, i.e. without involving communications between sets of processors.

Though the above operator Eqs. (3)-(6) is now of common use in GrSELA, ${ }^{56}$ we will here consider a slightly simplified collision scheme while relaxing the constraints Eqs. (4) and (5)

$$
\mathcal{C}(f)=\partial_{v_{\|}}\left\{\mathcal{D} \partial_{v_{\|}} f-\mathcal{V} f\right\}
$$

the operators $\mathcal{D}$ and $\mathcal{V}$ (see Fig. 1), respectively, modeling a diffusion and a drag

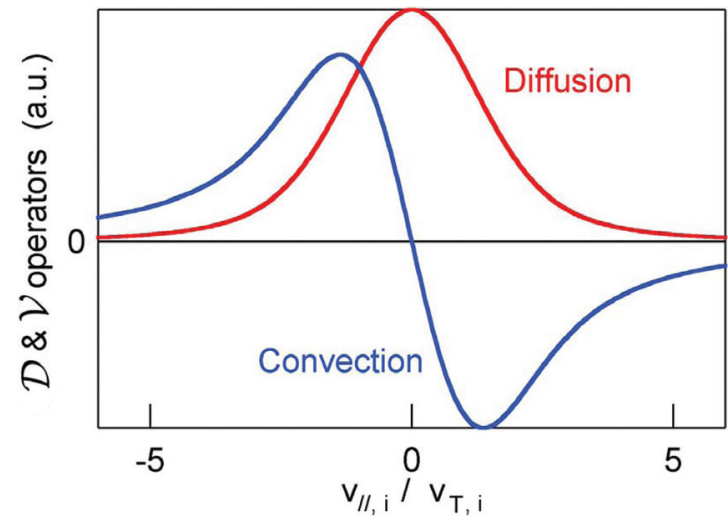

FIG. 1. (Color online) Parallel velocity dependance of the diffusive and convective operators $\mathcal{D}$ and $\mathcal{V}$ in Eqs. (10) and (11).

$$
\begin{gathered}
\mathcal{D}=3 \frac{\sqrt{\pi}}{2} \frac{v_{T}^{3} \epsilon^{3 / 2}}{q R_{0}} \nu_{\star} \frac{\Phi(v)-G(v)}{2 v} \\
\mathcal{V}=-\frac{v_{\|}}{v_{T}^{2}} \mathcal{D}
\end{gathered}
$$

Here $v_{T}=(T / m)^{1 / 2}$ denotes the thermal velocity, $q$ the safety factor, $R=R_{0}+r \cos \theta$ the major radius, and $\epsilon=r / R$ the inverse aspect ratio. The ion-ion collision frequency

$$
\nu_{i i}=\frac{4 \sqrt{\pi}}{3} \frac{n e^{4} \log \Lambda}{\left(4 \pi \epsilon_{0}\right)^{2} m^{2} v_{T}^{3}}
$$

is expressed in terms of the dimensionless ion-ion collisionality parameter $\nu_{\star}$ :

$$
\nu_{\star}=\frac{q R_{0}}{v_{T}} \frac{\nu_{i i}}{\epsilon^{3 / 2}}
$$

where $\epsilon_{0}$ is the permittivity of free space, $\log \Lambda \approx 17$ the Coulomb logarithm, and $n$ the ion density. Explicit expressions for Eqs. (10) and (11) involve the error function $\Phi$

$$
\begin{gathered}
\Phi(v)=\frac{2}{\sqrt{\pi}} \int_{0}^{v} e^{-x^{2}} \mathrm{~d} x \\
\Phi^{\prime}(v)=\frac{2}{\sqrt{\pi}} e^{-v^{2}}
\end{gathered}
$$

and the Chandrasekhar function $G$

$$
G(v)=\frac{\Phi(v)-v \Phi^{\prime}(v)}{2 v^{2}}
$$

Relaxing the constraints Eqs. (4) and (5) practically means when running a gyrokinetic code that $V_{\|}$and $T$ need not to be calculated at each time step and updated back into the collision scheme. Such an operator [Eq. (9)] thus introduces an additional friction in the parallel direction. A collisional entropy production rate can nonetheless still be computed ${ }^{21}$ which leads to a generalised force balance equation with a vanishing ion toroidal velocity and otherwise correct values of the neoclassical poloidal velocity, heat diffusivity, and collisional damping of axisymmetric flows, as would be calculated by a full Fokker-Planck operator. The above model, 
whilst preserving some attractive simplicity, thus allows for the exact recovery of the conventional features of neoclassical theory in the low (banana) and intermediate (plateau) tokamak-relevant collisionality regimes. Further discussion on this subject may be found in Sec. III.

The first piece in Eq. (9) accounts for a diffusion in parallel velocity $\mathcal{D}=\left\langle\Delta v_{\|}^{2}\right\rangle / 2$ generated by the Coulomb binary interactions. It is essentially responsible for the neoclassical diffusive transport and models the resonant enhancement of collisional effects in the presence of local trapping. The second piece is often referred to as a "dynamic friction" and is also reminiscent of the Fokker-Planck structure of the collision operator: $\mathcal{V}=\left\langle\Delta v_{\|}\right\rangle$. It is an average time rate of change of parallel velocity due to the scattering effects of collisions and accounts for the poloidal flow damping obtained from neoclassical theory.

Using Eq. (11), one can trivially see that the solution for the full- $f$ collisional problem is the local Maxwellian $f_{M}=f_{S M}\left(V_{\|}=0\right)$ for both the bulk equilibrium and the fluctuations, which allows one to write Eq. (9) in the compact form-to be compared to Eq. (3)

$$
\mathcal{C}(f)=\partial_{v_{\|}}\left\{\mathcal{D} f_{M} \partial_{v_{\|}}\left(\frac{f}{f_{M}}\right)\right\}
$$

$\mathcal{D}$ still being given by Eq. (10). The following Sec. III will discuss the physics both models Eqs. (3), (9), or (17) can address before assessing in Sec. IV their accuracy for computing neoclassical phenomena.

\section{B. A linearised $\delta f$ collision operator-best suited for Monte Carlo-based schemes}

Coulomb collisions in the full- $f$ XGC1 code are modeled by a well-known particle, momentum, and energy conserving linearised Monte-Carlo scheme, ${ }^{59-62}$ which uses the $\delta f$ concept of approximating by a Maxwellian the background field particles; the accuracy of the collision operator therefore degrades when the majority of the particles around the thermal energy are highly non-Maxwellian. Since XGC1 is a PIC code, each marker particle experiences the conventional energy-dependent Monte-Carlo scattering ${ }^{63}$ in energy and pitch-angle against the local Maxwellian background which is evaluated through a local averaging of the marker particles. The conservation properties of this scheme are ensured by adjusting the particle weights. ${ }^{59}$ In a full $-f$ simulation, these weights are invariant during the time advance process - corresponding to the Vlasov part of the FokkerPlanck equation-but are allowed to evolve during the collisional process.

The actual form of the Monte-Carlo collision operator used here pertains to the larger class of Lorentz collision operators, written in the pitch-angle coordinate $\lambda=v_{\|} / v$,

$$
\mathcal{C}(f)=\frac{\nu_{d}}{2} \frac{\partial}{\partial \lambda}\left(1-\lambda^{2}\right) \frac{\partial f}{\partial \lambda}
$$

where $\nu_{d}$ is the deflection collision frequency

$$
\nu_{d}=\frac{3}{2} \sqrt{\frac{\pi}{2}} \nu_{i i} \frac{\Phi(\bar{v})-G(\bar{v})}{\bar{v}^{3}}
$$

and $\nu_{i i}$ is the ion-ion collision frequency [Eq. (12)], $\Phi$ the error function [Eq. (14)], $G$ the Chandrasekhar function [Eq. (16)] and $\bar{v}=v / \sqrt{2} v_{T}$. The following Secs. III and IV complement the existing studies of neoclassical physics in the plasma edge $e^{64-66}$ whilst providing a successful cross-comparison between this Monte-Carlo scheme and the previously detailed Eulerian-based operators implemented in GyseLA.

\section{DISCUSSION: WHICH PHYSICS CAN WE ADDRESS?}

The physics one can address is usefully discussed in the framework of fluid theory. In the absence of sources of both momentum and particles, the momentum flux conservation reads:

$$
\begin{aligned}
m n \mathrm{~d}_{t} \mathbf{V}_{i}= & e n\left(\mathbf{E}+\mathbf{V}_{i} \times \mathbf{B}\right)-\nabla p_{i}-\nabla \cdot \overline{\bar{\Pi}} \\
& -m n \nu_{i e}\left(\mathbf{V}_{i}-\mathbf{V}_{e}\right),
\end{aligned}
$$

where subscript "e" (resp. "i") denotes electron (ion) quantities, and fluid (kinetic) quantities are written in uppercase (lowercase) letters. Writing $\overline{\overline{\mathbf{I}}}$ as the unit tensor, $\overline{\bar{\Pi}}=\int \mathrm{d}^{3} \mathbf{v} m$ $\left(\mathbf{v v}-\overline{\mathbf{I}}|\mathbf{v}-\mathbf{V}|^{2} / 3\right) f$ is the viscous stress tensor, which reduces at leading order to the parallel viscous stress. Focusing on the poloidally symmetric part of the latter equation, its equilibrium parallel projection simply reads

$$
e n E_{\|}-m n \mu_{i i}\left(V_{\theta i}-V_{\theta i}^{N C}\right)-m n \nu_{i e}\left(V_{\| i}-V_{\| e}\right)=0
$$

where $\langle\mathbf{b} \cdot(\nabla \cdot \overline{\bar{\Pi}})\rangle_{\theta} \approx m n \mu_{i i}\left(V_{\theta i}-V_{\theta i}^{N C}\right), V_{\theta i}^{N C}=K_{1} \frac{\nabla T}{e B}$ is the neoclassical prediction for the poloidal velocity, $\langle\cdot\rangle_{\theta}$ stands for the poloidal average, and $\mu_{i i}$ is the neoclassical ion viscous damping frequency, which can be approximated in the low-collisionality banana and plateau regimes ${ }^{67}$ by: $\mu_{i i} \approx 0.78 \sqrt{\epsilon} \nu_{i i} /\left(1+044 \nu_{\star}\right)$. Let us first concentrate on this term. We adopt the notation used in Ref. 68 , where $K_{1}$ primarily depends on the charge of the species, the aspect ratio and reverses sign depending on the collisionality regime. Practically, one correctly gets the poloidal flow damping as soon as the viscous stress tensor is correctly modeled. Physically, it means that both magnetic pumping due to the poloidal inhomogeneity of the magnetic field and Coulomb collisional interactions between trapped and passing particles are correctly accounted for. Both effects combine to enslave the ion poloidal velocity to the temperature gradient, or equivalently, to the ion diamagnetic velocity. This physics is an essential part of neoclassical theory and is intrinsically embedded in all model operators described here in Eqs. (3), (9), (17), or (18) which allow to exactly recover the neoclassical viscous stress tensor in the banana and plateau regimes—see e.g., details in Ref. 21.

Moving to the last term in Eq. (21), attention is drawn to the momentum-conserving properties of the collision operator. This friction term accounts for the inter species electron-ion transfer of momentum. Since momentum conservation between species is central in neoclassical theory, recovering conventional neoclassical equilibrium is delicate whilst the electron response remains adiabatic. In this case, the inter species friction needs to be replaced by a like-species friction: $m n \nu_{i i} V_{\| i}$, which tends to damp out the parallel 
flow. Provided Galilean invariance is maintained, as should be, the solution of the collisional problem is a shifted Maxwellian: $f_{S M}\left(v_{\|}\right)=f_{M}\left(v_{\|}-V_{\| i}\right)$ and the correct amount of parallel friction forces is accounted for. The collision schemes Eqs. (3) and (18) have been coined so as to allow for the expected momentum and energy conservation properties. From a practical point of view, this approach consists in performing the collisions locally, in the rest frame of the moving ions. It also means that when running a gyrokinetic code, $V_{\| i}$ and $T$ must be calculated at each time step and fuelled back into the collision operator.

Interestingly though, even if crucial for the correct modeling of parallel friction forces, accurate satisfaction of Galilean invariance has little influence (i) on the radial structure and the magnitude of the poloidal flows which depend on the modeling of the viscous stress tensor and (ii) on the heat transport, as demonstrated in Refs. 21 and 62 and Figs. 3-6. A simplified collision operator such as the one in Eq. (9) or (17) thus also accurately models the collisional radial cross-field heat transport, as a complete Fokker-Planck operator would. It would not be able though to account for the correct amount of parallel viscosity for it introduces an additional friction force in the toroidal direction which would lead to (i) overestimating the momentum loss of circulating ions and electronsespecially in the banana regime, thus underestimating the bootstrap current while also (ii) overestimating the particle fluxes. ${ }^{62}$ This would be of major concern while modeling multiple species and accounting for inter-species friction; on the other hand, the simplifying approach of Eq. (9) or (17) may accordingly be appropriate to modeling the neoclassical equilibrium in the case of an adiabatic electron response.

This point is easily understood while looking at the transverse equilibrium of Eq. (20) - the classical radial force balance equation:

$$
E_{r}-v_{\varphi} B_{\theta}+v_{\theta} B_{\varphi}=\frac{\nabla p}{n e} .
$$

Neoclassically, poloidal rotation $v_{\theta}^{N C}=K_{1} \nabla T / e B$ is tied to the temperature gradient, as verified in Figs. 3 and 4. On the other hand, the accurate computation of the self-consistent radial electric field is a known central problem for full $f$ gyrokinetics, ${ }^{21,69}$ part of it being due to the following degeneracy, intrinsic in neoclassical theory: prediction in an axisymmetric system is on the combination $E_{r}-v_{\varphi} B_{\theta}$, which is degenerate in the absence of toroidal symmetry-breaking mechanisms, should they be (i) of geometric nature -ripple, ${ }^{70,71}$ error fields - or should they appear (ii) through the onset of the turbulence $-\mathbf{E} \times \mathbf{B}$ shear, ${ }^{72}$ turbulent Reynolds stresses, ${ }^{73-75}$ or intensity gradients- or (iii) through the inclusion of sources of toroidal momentum. The restriction of the conventional neoclassical equilibrium in Eq. (9) to its sub-class associated with a vanishing mean toroidal velocity therefore allows to relax this degeneracy and unambiguously relate the radial electric field to the poloidal rotation and the thermodynamic forces whilst accurately modeling the exact cross-field transport and flow generation mechanisms in the radial-poloidal plane as a more general Galilean-invariant Fokker-Planck operator would. On the other hand, this sim- plified collision operator is of course not suitable for addressing the important question of the generation of toroidal momentum - the observed spontaneous toroidal spin-up. ${ }^{76-78}$ In that case, the Galilean-invariant versions Eqs. (3) and (18) are required; a dedicated study of intrinsic rotation with both GYSELA and XGC1 is to be reported elsewhere. ${ }^{79,80}$

A careful accounting of collisions is also important to correctly describe the residual level of the low frequency turbulence-induced axisymmetric sheared $\mathbf{E} \times \mathbf{B}$ mean and zonal flows. The importance of such flows, linearly damped through collisions alone and which dynamically participate in turbulence self-regulation has long been emphasised. ${ }^{25}$ Collisions may thus impact the level of turbulent transport, especially close to marginality, ${ }^{2,28-30}$ in regimes were turbulent transport is expected to be most sensitive to $\mathbf{E} \times \mathbf{B}$ flow regulation. A strong commitment for any collision operator is thus to accurately model the collisional damping of such flows ${ }^{27}$ as shown in Fig. 8 .

From a terminological point of view, the "mean" field of quantity $\mathrm{x}$ stands throughout this paper for the temporal average $\langle x\rangle_{t}$ over a collision time (or above), in a statistical steady state with a constant drive of the turbulence, i.e., with a constant heat flowing across the system. Mean fields are of course profiled both in real and velocity spaces. Inversely, any quantity $x-\langle x\rangle_{t}$ which is dynamically faster than a collision time will be called "zonal". Especially, mean and zonal flows are such that: $x \equiv \partial_{r} \phi_{00}$.

Note that the above distinction is largely irrelevant from an experimental point of view: the distinction between "zonal" and "mean" flows is indeed based on the possibility to record a temporal series of instantaneous flow profiles, with a sampling frequency well below the ion-ion collision time. In other words, one needs both a fine enough spatial and fast enough temporal resolution to disentangle the "mean" from the "zonal" part in an actual measurement. These are constraints difficult to match with current experimental apparatuses; "zonal flows" thus indistinctly refer in experiments to both the "mean" and the "zonal" parts in the above sense. However difficult to disentangle experimentally, this distinction is easily made in a modeling activity. It is also of prime importance in modeling as both components are likely driven by different physical mechanisms.

From a physics perspective, existence of a perennial non-vanishing "mean" quantity with a nontrivial radial profile and self-consistent turbulence-induced evolution of the background mean profiles are two sides of the same coin. Both approaches translate the underlying dynamics that happens at intermediate meso-scales. Therefore, the ability to describe self-organisation at meso-scales and larger is the more relevant problem. ${ }^{1}$ It is an acknowledgment of the fact that a tokamak is indeed an open system in which self-organisation plays a dominant role. This translates numerically in self-consistently evolving the mean profiles [the full distribution function of the system] due to the combined action of the turbulence, of the neoclassical dynamics and of an external distribution of sources and sinks. Much debate has lately been triggered comparing the relative merits of the so-called "flux-tube," " $\delta f, "$ and "fixed gradient" approaches to socalled "global," "full-f," and "flux-driven" models. A further 
detailed discussion of these topics can be found in a separate publication. ${ }^{49}$

At last, let us emphasise that the collisional systems described here do intrinsically satisfy an H-theorem, as shown from an entropy extremalisation principle ${ }^{21}$ for operators Eqs. (3), (9), and (17). Due to this entropy principle, the full distribution function is guaranteed to relax towards a Maxwellian whilst most model operators only act on the perturbed distribution function, making the approaches here most appropriate when calculating the complete collisional equilibrium, including both the perturbed and the unperturbed problem. The net positive entropy production rate mostly originates from the dissipation processes occurring at the interface between the trapped and the passing regions in phase space. As a diffusive boundary layer, this interface gets increasingly localised as $\nu_{\star}$ diminishes. Within this layer, the dynamics of the collisions is accurately modeled by a friction force between the trapped and the untrapped particles with a favoured direction along the magnetic field lines; a qualitative discussion in this respect may be found in Fig. 7.

In summary, operator Eq. (9) or (17) is not suited for studying the generation of parallel momentum whereas operators Eqs. (3) and (18) are. All operators Eqs. (3), (9), (17), and (18) on the other hand accurately describe (i) the collisional damping of the mean and the zonal flows, (ii) the neoclassical heat transport processes and (iii) the plasma poloidal rotation in the banana and plateau regimes whilst remaining numerically efficient.

\section{RECOVERING THE NEOCLASSICAL RESULTS}

An accurate calculation of the radial electric field is of prime importance in full- $f$ computations, ${ }^{48}$ especially so as not to generate artificial equilibrium flows which could hinder the onset of the neoclassical equilibrium. It is therefore important, as preliminary tests, to appraise the system's behaviour with regard to simple constrained tests: let us consider the following simplest yet non trivial equilibrium state of the collisional gyrokinetic problem

$$
\begin{gathered}
f_{0}=\frac{\mathcal{N}}{\left(2 \pi T_{0} / m\right)^{3 / 2}} e^{-H / T_{0}} \\
\frac{e \phi_{0}}{T_{0}}=-\log \left(\frac{n_{0}}{\mathcal{N}}\right)+c_{1} K_{0}+c_{2} I_{0}
\end{gathered}
$$

Here $H=m v_{\|}^{2} / 2+\mu B+e \phi_{0}$ is the hamiltonian, $\mathcal{N}$ is a constant, so is $T_{0}$ the temperature, $\phi_{0}$ is the initial (and equilibrium) electric potential depending on the radial coordinate only, $n_{0}$ is the density profile, $K_{0}$ and $I_{0}$ are the modified Bessel functions of the first type and $c_{1}$ and $c_{2}$ are complex coefficients such that $\phi_{0}$ vanishes at the radial boundaries. This special choice Eq. (23) is obviously both (i) a motion invariant and (ii) satisfies $\mathcal{C}\left(f_{0}\right)=0$, henceforth being a general solution of the collisional gyrokinetic system. In the case of a constant temperature $T_{0}$ the usual force balance Eq. (22) trivially reduces to: $e \nabla \phi / T_{0}=-\nabla n / n$ : the electric field balancing the density gradient. When initialising Grsela with Eqs. (23) and (24) we indeed find that the long-time stationary equilibrium solution for the electric field is as expected Eq. (24) within a few percent precision, validating the stability of the computation. Further checks have included assessing the sensitivity of the results to the choice of the initial state: either a local Maxwellian or a canonical Maxellian: ${ }^{46-48}$ as should be, no dependence other than transient is found, the same statistical results being robustly obtained from different initialisations. In the remainder of this paper, GyseLA is initialised with a canonical Maxwellian and XGC1 with a local Maxwellian.

Neoclassical theory is tested whilst running below the linear ITG instability threshold: $R / L_{T}=3$ and $R / L_{n}=2.2$, where $L_{x}=-x / \nabla x$ is the gradient length for quantity $\mathrm{x}$. As a result, all the simulations reported in this section do not exhibit any turbulence nor turbulent transport. Other parameters are representative of core tokamak plasmas and locally read at mid radius $\quad r=0.5 a: \quad \epsilon=r / R=0.17, \quad T_{i} / T_{e}=1, \quad q=1.4 \quad$ and $s=(r / q) d q / d r=0.78$. In the current section, the neoclassical problem is axisymmetric and the grid size respectively involves (i) for GYSELA at $\rho_{\star}=\rho_{i} / a=1 / 256$ over $10^{9}$ grid points on a half-torus $\left(r, \theta, \phi, v_{\|}, \mu\right)=(256,256,8,128,16)$ mesh and (ii) above $1.025 \times 10^{9}$ particles on 10258 processors for XGC1 at $\rho_{\star}=1 / 184$. Here, $a$ is the minor radius, $\epsilon$ the inverse aspect ratio, $T_{i}$ and $T_{e}$ the ion and electron temperatures, $q$ the safety factor and $\rho_{i}$ the ion Larmor radius.

The ability of full- $f$ codes to accurately compute the mean radial electric field $E_{r}$-differently speaking, the long wavelengths of the electric potential $\phi$-has recently been questioned. ${ }^{69}$ Figure 2 shows an accurate recovery of the radial force balance Eq. (22) in both approaches, on the basis of conventional first order gyrokinetic equations, regardless of differences in the boundary conditions and details of the temperature profiles. This behaviour robustly holds in either neoclassical or turbulent regimes, for all investigated collisionalities spanning throughout the banana and plateau regimes. The poloidal velocity is here calculated by two means: (i) by means of the force balance: $\nabla p / n e-E_{r}$ $+v_{\varphi} B_{\theta}$, each of the latter terms being independently plotted and (ii) self-consistently within GySELA: $v_{\theta}^{\text {GYS }}$ or XGC1: $v_{\theta}^{\mathrm{XGC}}$ from the distribution function as the sum Eq. (25) of the poloidal components of the $\mathbf{E} \times \mathbf{B}$ Eq. (A7), grad-B Eq. (A8), curvature Eq. (A9), and parallel flows in addition to the curl of the magnetisation Eq. (26):

$$
\begin{gathered}
v_{\theta}=\frac{1}{n} \iint m^{2} B_{\|}^{*} \mathrm{~d} v_{\|} \mathrm{d} \mu\left(\mathbf{v}_{\mathbf{E} \times \mathbf{B}}+\mathbf{v}_{\nabla B}+\mathbf{v}_{c}+v_{\|} \mathbf{b}\right) \cdot \mathbf{e}_{\theta} f \\
+\mathbf{v}_{m a g} \cdot \mathbf{e}_{\theta} \\
\mathbf{v}_{m a g}=\frac{1}{n e} \nabla \times\left(\mathbf{b} \iint m^{2} B_{\|}^{*} \mathrm{~d} v_{\|} \mathrm{d} \mu \mu \bar{f}\right)
\end{gathered}
$$

Here $\mathbf{e}_{\theta}$ denotes the unit vector in the poloidal direction. The response of the electric potential, which always adjusts so as to satisfy Eq. (22), is as expected significantly different in the banana Fig. 2(a) and the plateau regimes Figs. 2(b) and 2(c) since the poloidal velocity itself strongly depends on $\nu_{\star}$ (see Fig. 3 below). 
In the banana regime, the radial electric field mainly compensates the density gradient, whereas in the plateau regime, the poloidal velocity is smaller and the radial electric field increases so as to mainly compensate the pressure gradient. Hence the different behaviours observed for $E_{r}$ in Figs. 2(a), 2(b), and 2(c), whilst the global force balance remains satisfied within a few percent precision. Also, the small discrepancy noted in Fig. 2(c) between $\rho=0.2$ and
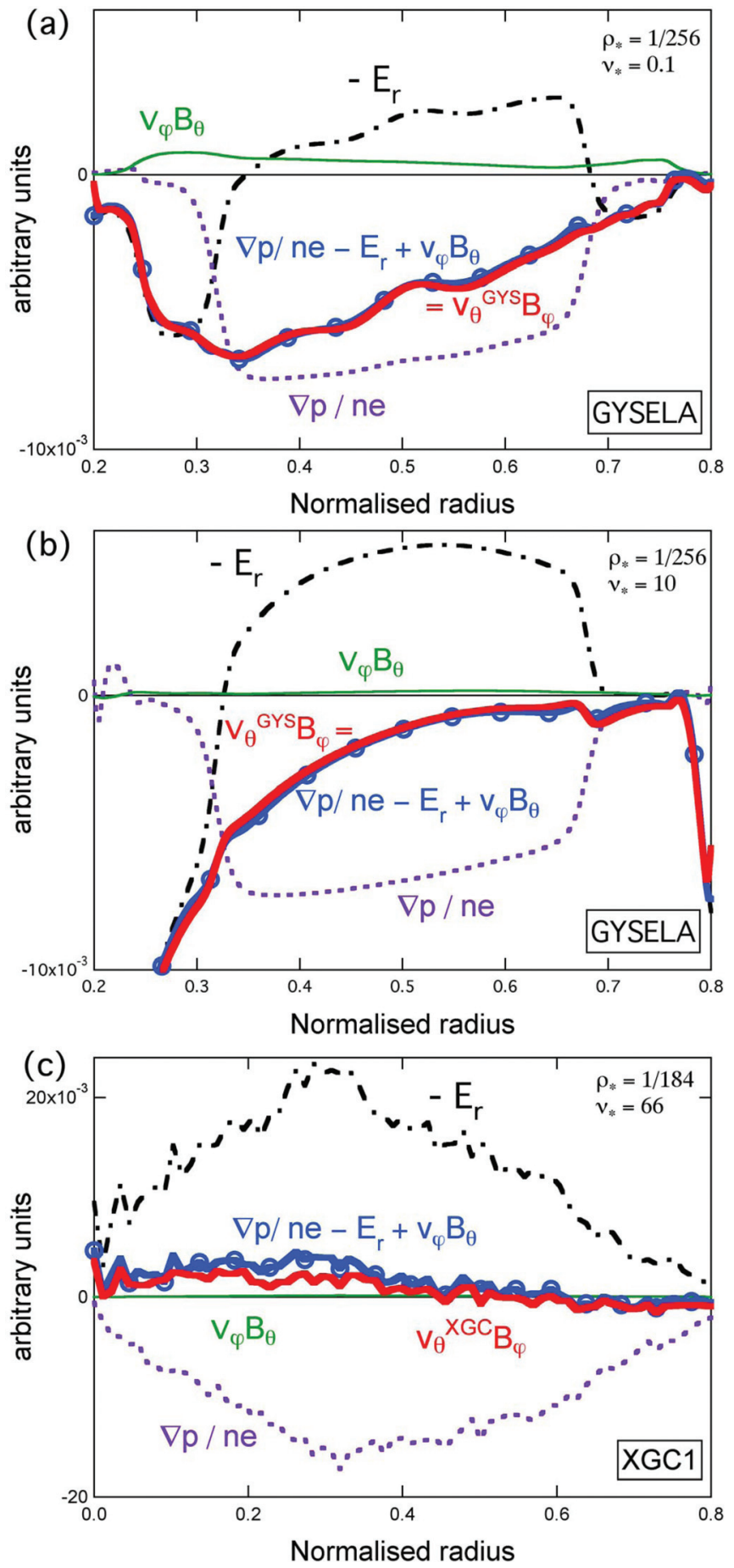

FIG. 2. (Color online) Test of the accuracy of the force balance equation (22) in the banana $\nu_{\star}=0.1$ (a) and the plateau $\nu_{\star}=10$ and 66 (b) - (c) regimes. The poloidal velocity is evaluated by two means: either consistently evolved within Gysela: $v_{\theta}^{G Y S} B_{\varphi}$ or $\mathrm{XGC} 1: v_{\theta}^{X G C} B_{\varphi}$ or as the sum: $\nabla p / n e-E_{r}+v_{\varphi} B_{\theta}$. Excellent agreement is found, regardless of the precise moment in the simulation.
0.4 in normalised radius may be explained as the combination of both the higher collisionality: at smaller "banana" collisionalities a closer satisfaction of the radial force balance is found and of the nature of Fig. 2(c) as an instantaneous snapshot: a time averaged equivalent plot would further smooth out this discrepancy as the Monte Carlo fluctuations would be reduced. Shown here is thus an upper-bound limit of the instantaneous deviation from radial equilibrium.

The neoclassical prediction $v_{\theta}=K_{1}\left(\nu_{\star}, \epsilon\right) \nabla T / e B$ is essentially twofold: the analytical prediction on $K_{1}$ is (i) accurate in the asymptotic banana and Pfirsch-Schlüter regimes, whilst only approximate in the plateau regime; also, regardless of the precise transition between regimes (ii) $v_{\theta}$ should reverse sign with the collisionality: it is predicted to rotate in the electron diamagnetic direction in the PfirschSchlüter regime and in the ion diamagnetic direction in the banana regime. ${ }^{68} \mathrm{~A}$ consistent agreement with this prediction is found with both GrseLA and XGC1 in the asymptotic banana regime in which this prediction is valid; the rotation reversal as collisionality increases is also confirmed ${ }^{81}$ with a transition from the banana to the plateau regime around $\nu_{\star}^{b-p} \approx 1$. Nonetheless, the theory in Ref. 68 is rather inaccurate in predicting the critical value $\nu_{\star}^{c}$ for which the ion poloidal rotation vanishes on average; a quantitative comparison to our results is therefore impossible in the plateau regime. In the case of a large radial electric field shear, the poloidal flow velocity is well-known to possibly increase well above these neoclassical values and become a significant fraction of the ion diamagnetic velocity. ${ }^{82}$ This orbit squeezing correction is plotted in Fig. 3 [green (small squares) curve] but since the radial electric shear in all simulations is low, its effect is here subdominant.

A widely used expression for the poloidal rotation in the banana regime is $v_{\theta}=1.17 \nabla T / e B$. This result is indeed the neoclassical prediction in the limit of infinite aspect ration tokamaks $\epsilon \rightarrow 0$. At intermediate values of $\epsilon$, it should be noted that a more accurate prediction exists in the banana

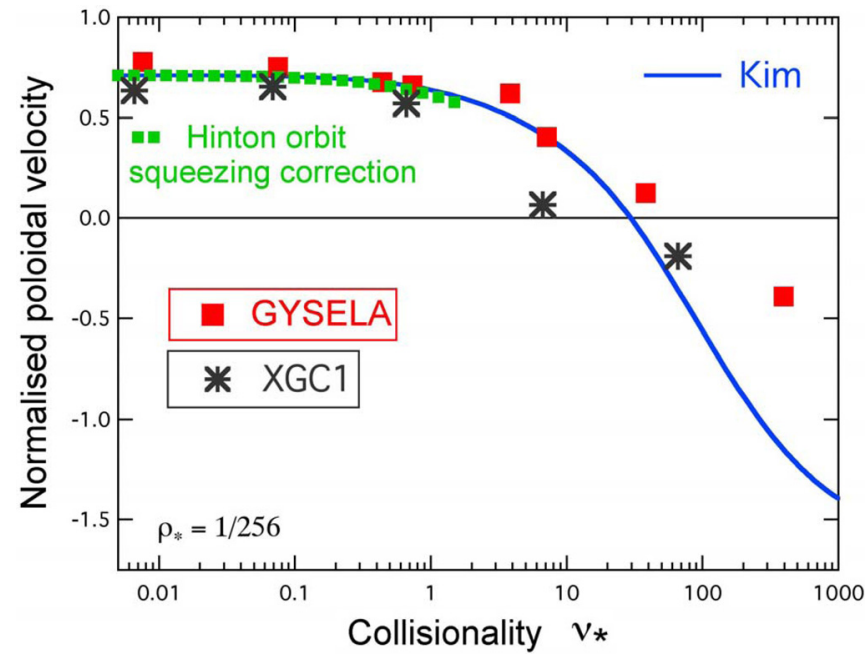

FIG. 3. (Color online) Neoclassical poloidal velocity normalised to the temperature gradient (coefficient $K_{1}$ in Ref. 68) as a function of the collisionality $\nu_{\star}(\epsilon=0.17$ and $\theta=\pi)$. The definition of the collisionality parameter in Ref. 68 is slightly different to the one we use here and has thus been renormalised to the value in Eq. (13). This result is robustly obtained for various $\rho_{\star}$ values. 


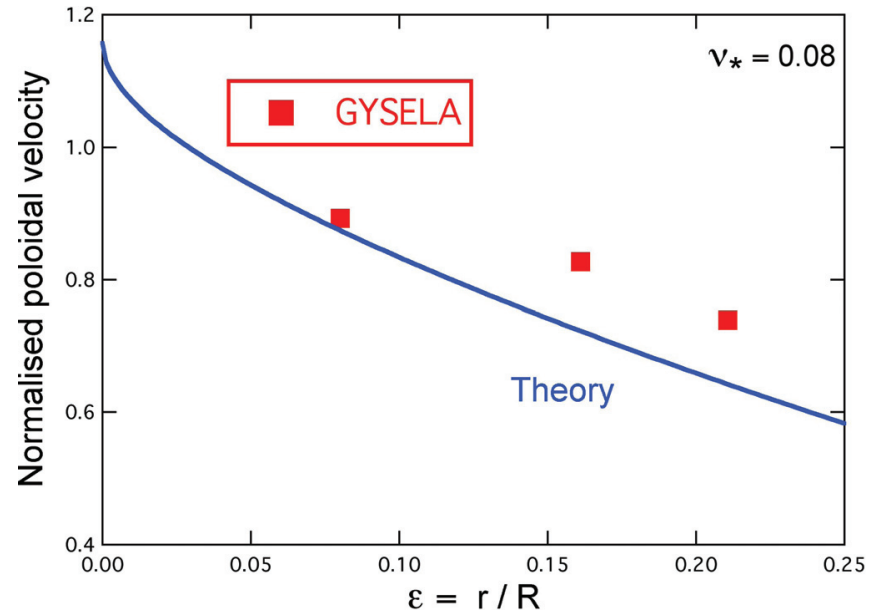

FIG. 4. (Color online) Neoclassical poloidal velocity normalised to the temperature gradient (coefficient $\left.K_{1}\right)$ as a function of $\epsilon\left(\nu_{\star}=0.08\right)$.

regime, ${ }^{68}$ as shown in Fig. 4 and that the 1.17 pre-factor can be significantly modified.

To investigate this, we performed three different neoclassical simulations at $\nu_{\star}=0.08$ corresponding to three different values of the parameter $\epsilon$. The results show a good agreement with the neoclassical prediction, confirming the significant importance of the aspect ratio dependence in the evaluation of the neoclassical poloidal rotation.

This parameter also influences transport, as displayed in Fig. (5). The same three simulations at $\nu_{\star}=0.08$ are displayed in Fig. 5. They show the normalised ion heat diffusivity $\overline{\mathcal{L}_{i}}$ (Ref. 83) at five different radii plotted against $\epsilon$,

$$
\overline{\mathcal{L}_{i}}=\frac{\chi_{i}}{2 \chi_{b}}
$$

where $\chi_{b}$ reads: $\chi_{b}=\left(m v_{T} / e\langle B\rangle\right)^{2} q v_{T} \nu_{\star} / R_{0}, v_{T}=(T / m)^{1 / 2}$ and $\langle B\rangle$ is flux-surface averaged. Whilst the reduced Hirshman-Sigmar operator ${ }^{84}$ underestimates the ion energy flux by about $10 \%$ in all aspect ratio regimes, we confirm ${ }^{81}$ that in the intermediate aspect ratio regime the widely used Chang-Hinton model ${ }^{85}$ overestimates by about $25 \%$ the ion thermal diffusivity $\chi_{i}$. A good agreement is found with the more accurate Taguchi ${ }^{83}$ banana regime model.

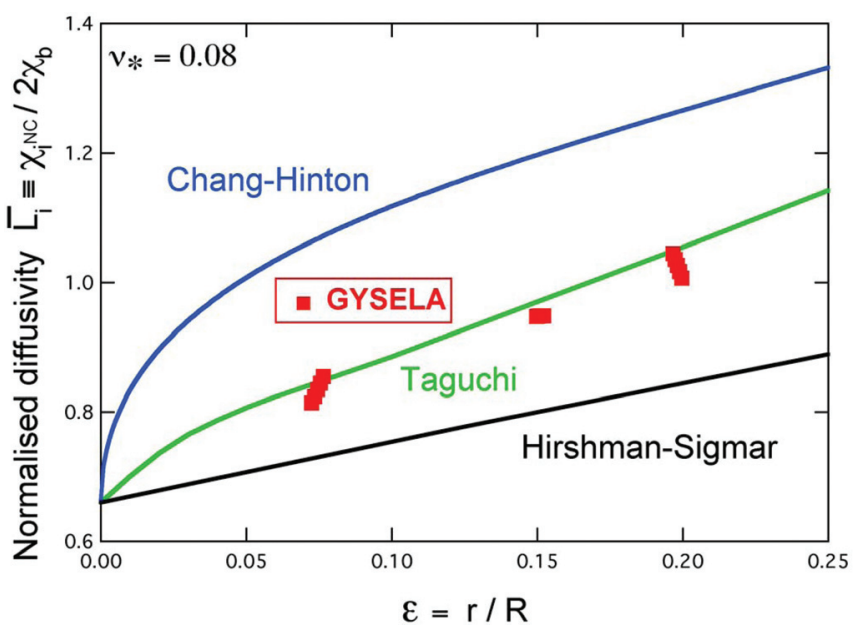

FIG. 5. (Color online) Normalised neoclassical ion heat diffusivity (coefficient $\overline{\mathcal{L}})$ as a function of $\epsilon\left(\nu_{*}=0.08\right)$.
At low values of $\epsilon$, the ion thermal diffusivity as derived by Chang and Hinton ${ }^{86}$ provides an interpolation between the banana, plateau and Pfirsch-Schlüter regimes in the limit of a vanishing impurity contribution and no Shafranov shift:

$$
\begin{aligned}
\frac{\chi_{i}^{C H}}{\rho_{i}^{2} \omega_{c}}= & 2 \sqrt{2} \frac{q v_{T}}{\omega_{c} R} \nu_{\star}\left\{\frac{066+188 \sqrt{\epsilon}-1.54 \epsilon}{1+1.03 \sqrt{\nu_{\star}}+0.31 \nu_{\star}} I_{b-p}\right. \\
& \left.+\frac{0.59 \epsilon \nu_{\star}}{1+0.74 \epsilon^{3 / 2} \nu_{\star}}\left(I_{b-p}-I_{P S}\right)\right\} .
\end{aligned}
$$

The $2 \sqrt{2}$ coefficient comes from our definition of the thermal velocity: $v_{T}=(T / m)^{1 / 2}$. This relation Eq. (28) is displayed in Fig. 6. A good agreement is found with both Gysela and XGC1 throughout the entire banana and plateau regimes.

The $I_{b-p}$ term represents the neoclassical contributions from the banana and plateau regimes and describes the transition between them: $I_{b-p}=1+3 \epsilon^{2} / 2$. Similarly, the $I_{P S}$ term represents the Pfirsch-Schlüter contribution when $\nu_{\star} \epsilon^{3 / 2}>1$ : $I_{P S}=\left(1-\epsilon^{2}\right)^{1 / 2}$. At finite aspect ratio $(\epsilon=0.17)$, the discrepancy observed at low collisionalities between the GySELA results and the Chang-Hinton prediction is consistent with the above discussion in Fig. 5; we again find that at intermediate aspect ratios, the Taguchi model is more accurate in the banana regime.

As compared to early classical theories ${ }^{87}$ in which the spatial variation of the magnetic field has no influence on the transport, neoclassical theory is deeply connected to resonance phenomena that occur through particle trapping in inhomogeneities of the magnetic field. Collisions contribute to regularising the trapping singularities through a broadening of the otherwise highly localised region of phase space which delineates the trapped region from the passing. The broadening of this region with increasing collisionality is displayed in Fig. 7.

Due to the spatial nonuniformity of the magnetic field, iso-contours of the distribution function display the typical "cat-eye" shape in phase space, revealing particle trapping. Starting from the canonical Maxwellian, ${ }^{48}$ the initial

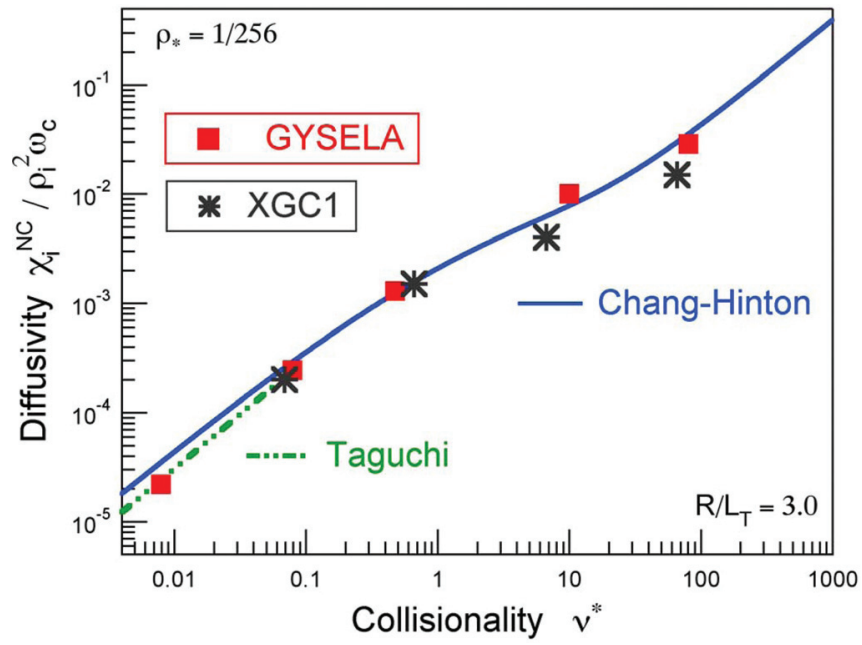

FIG. 6. (Color online) Neoclassical heat diffusivity normalised to its Bohm value as a function of collisionality $\nu_{\star}(\epsilon=0.17)$. 


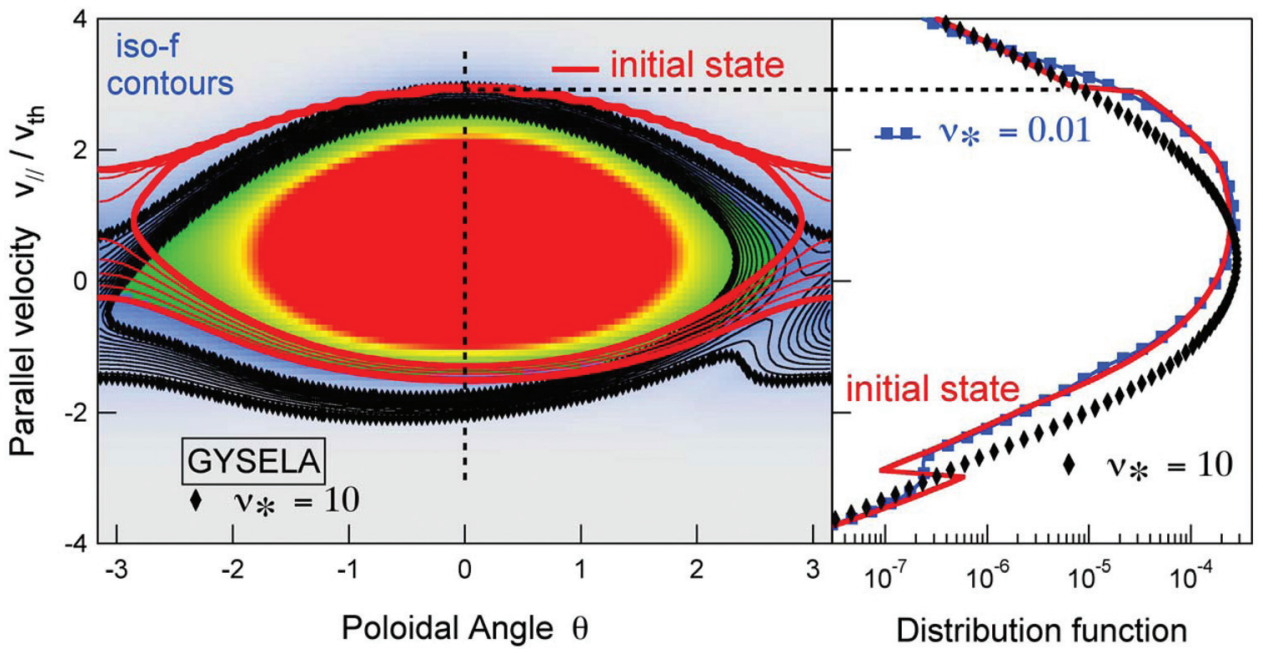

FIG. 7. (Color online) Collisional regularisation of the full distribution function at the boundary layer between the trapped and untrapped regions of phase space. This regularisation is essentially occuring along the magnetic field lines. The island in $\left(\theta, v_{\|}\right)$space represents isocontours of the full distribution function. distribution function is a motion invariant, i.e., an exact solution of the collisionless gyrokinetic equation but not a solution of the collisional problem. It is singular at the vicinity of the trapping boundary (sometimes also referred to as the "loss cone"), as clearly illustrated by the transverse plot of the distribution function at $\theta=0$ (red curve). The dissymmetry in $v_{\|}$comes from the choice of the canonical Maxwellian. Let us concentrate on this right-hand side of Fig. 7. The two simulations at $\nu_{\star}=10$ and $\nu_{\star}=0.01$ are compared at the same time, i.e., after respectively 10 collisions and 0.1 collision. As expected, as the collisionality increases the full distribution function (i) is increasingly regularised and (ii) relaxes towards a local Maxwellian. The form of the distribution function, while relaxing (blue squares), achieves a compromise between a function of the motion invariants (black diamonds) and a local Maxwellian (solid red). On the iso-contour plot, as best seen close to $\left(\theta, v_{\|} / v_{T}\right)=(0,3)$, the initial highly localised region delineating the trapped domain from the untrapped broadens as collisionality increases (diamond contours).

This regularisation occurs predominantly transversely to the island, providing a heuristic argument as to why the correct neoclassical equilibrium could be accurately recovered in collision operators Eqs. (3), (9), or (17) without a transverse

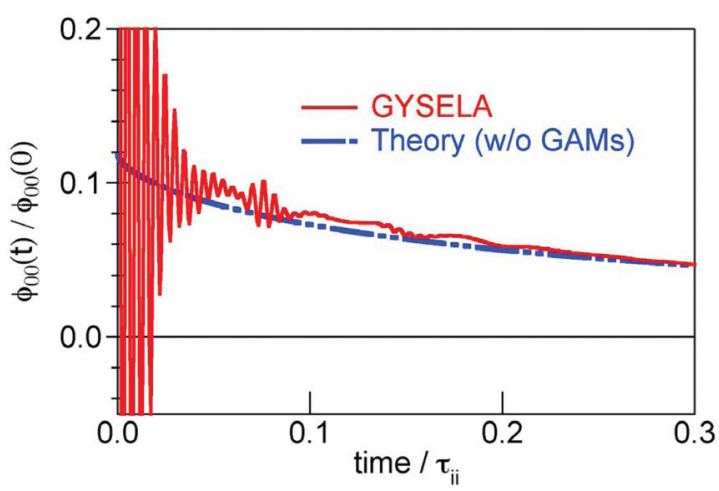

FIG. 8. (Color online) Collisional damping of the mean and the zonal flows confronted to the analytical prediction by Hinton and Rosenbluth (Ref. 27) without the GAM contribution $\left(\nu_{\star}=0.04, \epsilon=0.18\right)$. The frequency obtained with GYSELA agrees within the percent with the precise GAM frequency calculation (Ref. 88). diffusion operator (advection in $v_{\|}$only). It connects to the well-known result ${ }^{22}$ that in the banana regime this boundary layer provides the dominant contribution to the heat transport coefficient. At the root of neoclassical transport, a perturbed hamiltonian-i.e., particle trapping-enhances collisional transport due to the resonant interaction ${ }^{21}$ occurring in this region between the particles and this perturbation. A correct modeling of this boundary layer is therefore the crucial piece of physics which allows to recover the correct neoclassical transport displayed in Fig. 6.

At last, Rosenbluth and Hinton predicted ${ }^{26}$ the existence of a linearly undamped axisymmetric flow $\phi_{00}$ which survives the collisionless linear Landau damping process. With collisions though, this residual flow is slowly damped, ${ }^{27}$ mainly through the collisional friction the trapped particles exert on the passing particles at the vicinity of the trapping boundary. A $\phi_{00}$ initial perturbation is followed in time in Fig. 8. The plasma parameters are those in Ref. 27: $\nu_{\star}=0.04, \epsilon=0.18$, and $q=1.4$ at mid radius. The observed decay accurately matches the expected analytical damping rate; in the analytical model though, time scales are larger than the ion bounce period so that the transient oscillations displayed by the gyrokinetic result are not modeled. A Fourier transform of these oscillations shows again as expected a very close agreement $(<1 \%)$ with the Geodesic Acoustic Mode frequency. ${ }^{88}$

\section{CONCLUSION}

The precise description of neoclassical theory is an important step in the process of modeling the plasma dynamics as it provides the system with an ever-existing background cross-field transport, a means to satisfy an H-theorem, a linear flow damping mechanism for axisymmetric flows, an effective "return force towards Maxwellianity" and strong constrains on plasma rotation. The important open question of how the slow neoclassical equilibrium may interplay with the faster, smaller-scale turbulence as the system self-organises is still unsettled. To this end, it is key to design, implement, and carefully test collision operators acting upon the full gyrokinetic distribution function of the system so that the latter interplay may occur, fully consistently. ${ }^{49}$ 
A set of such operators has been presented, discussed and thoroughly verified in the course of this paper for as different numerical architectures as the Eulerian-like structure of GYSELA and the Lagrangian (PIC) structure of XGC1. In both cases, as a special mandatory feature of full- $f$ codes, without an external forcing, the full distribution function is designed to relax towards a Maxwellian. Numerically speaking, both approaches are appreciably different: whilst the latter is well suited for Monte-Carlo processes and written in the more usual pitch-angle and energy variables, the former features an efficient parallelisation with respect to the adiabatic variable and is gridded in the parallel velocity only. As such it takes advantage of a fixed grid discretisation as present in Eulerian or semi-Lagrangian approaches whilst avoiding much of the parallelisation cost inherent to more general operators.

The essential results of neoclassical theory are reproduced in both cases, providing an interesting cross-validation of these two approaches. In particular, the neoclassical transport and poloidal rotation with their collisionality and aspect ratio dependence, along with the collisional mean and zonal flow damping are accurately reproduced. Physically, it means that the correct amount of friction forces between trapped and passing particles at the trapping boundary is accounted for. These forces dominantly occur close to the separatrix, transverse to the magnetic island, and in a direction that is almost aligned with the magnetic field lines. As an illustration, regularisation of the distribution function, singular at the trapping boundary without collisions, is shown in the parallel direction.

Forthcoming work will focus on the interplay between the slow, large-scale neoclassical equilibrium, the fast, small-scale turbulence whilst the thermodynamic forces acting upon the system can self-consistently evolve given an external distribution of sources and sinks, comparably to what happens in experiments. Pushing the envelope in these matters especially means providing answers to the following open questions: (i) what will the flow patterns look like in flux-driven statistical steady-state? (ii) how may one characterise an attracting fixed point for the dynamics? A followup on these will appear shortly.

\section{ACKNOWLEDGMENTS}

The authors want to thank Ch. Passeron for constant help in the development of the Gysela code and to acknowledge fruitful discussions with S.J. Allfrey, O.D. Gürcan, J. Kwon, and C.J. McDevitt as well as crucial interactions at the Festival de Théorie in Aix-en-Provence, France, 2007 and 2009. This work, supported by the European Communities under the contract of Association between EURATOM and CEA, was carried out within the framework of the European Fusion Development Agreement. The views and opinions expressed herein do not necessarily reflect those of the European Commission. This work also benefited from the support of U.S. SciDAC GPS-TTBP, Grant No. DE-FC02-08ER54959 and from the Ministry of Education, Science and Technology of Korea via the WCI project 2009-000; it was performed using HPC resources from NCCS Jaguar and GENCI-[CCRT/CINES/IDRIS], Grant No. 2009-052224.

\section{APPENDIX: STANDARD FULL-F GYROKINETIC FORMULATION}

The electrostatic branches_-slab, toroidal, and trapped modes-of the ITG instability are modeled whilst solving the coupled system of the collisional gyrokinetic equation

$$
\begin{aligned}
& \frac{\partial \bar{f}}{\partial t}+\left(\mathbf{v}_{\mathbf{E} \times \mathbf{B}}+\mathbf{v}_{\nabla B}+\mathbf{v}_{c}\right) \cdot \nabla \bar{f}+v_{\|} \nabla_{\|} \bar{f}+\frac{\mathrm{dv}_{\|}}{\mathrm{dt}} \partial_{v_{\|}} \bar{f} \\
& \quad=\mathcal{S}(\bar{f})+\mathcal{C}(\bar{f})
\end{aligned}
$$

and quasi-neutrality equation

$$
\begin{gathered}
\frac{e}{T_{e}}[\phi-\langle\phi\rangle]-\frac{1}{n_{e q}} \nabla_{\perp} \cdot\left[\frac{m n_{e q}}{e B^{2}} \nabla_{\perp} \phi\right] \\
=\frac{1}{n_{e q}} \iint \frac{2 \pi B_{\|}^{*}}{m} \mathrm{~d} v_{\|} \mathrm{d} \mu\left(\bar{f}-\bar{f}_{\text {init }}\right)
\end{gathered}
$$

for any gyroaveraged full ion distribution function $\bar{f}$ and electrostatic potential $\phi$. The evolution equations of the gyrocentre coordinates classically read

$$
\begin{gathered}
\frac{\mathrm{d} \mathbf{x}}{\mathrm{d} t}=v_{\|} \mathbf{b}^{*}+\mathbf{v}_{\mathbf{E} \times \mathbf{B}}+\mathbf{v}_{\nabla B}+\mathbf{v}_{c} \\
m \frac{\mathrm{d} v_{\|}}{\mathrm{d} t}=-\mu \nabla_{\|} B-e \nabla_{\|} \bar{\phi}+\frac{m v_{\|}}{B} \mathbf{v}_{\mathbf{E} \times \mathbf{B}} \cdot \nabla B
\end{gathered}
$$

where

$$
\begin{gathered}
\mathbf{b}^{*}=\frac{\mathbf{B}}{B_{\|}^{*}}+\frac{m v}{e B_{\|}^{*}} \frac{\mu_{0} \mathbf{J}}{B} \\
B_{\|}^{*}=B+\frac{m v_{\|}}{e B} \mu_{0} \mathbf{b} \cdot \mathbf{J}
\end{gathered}
$$

and $\mu_{0} \mathbf{J} / B=\nabla \times \mathbf{b}-\mathbf{b} \times \nabla B / B$, with $\mathbf{b}=\mathbf{B} / B$. The parallel and transverse gradients are respectively: $\nabla_{\|}=\mathbf{b}^{*} \cdot \nabla$ and $\nabla_{\perp}=\nabla-\nabla_{\|} \mathbf{b}^{*}$. The three drift velocities evaluated at the gyrocentre coordinates-respectively the $\mathbf{E} \times \mathbf{B}$, grad-B, and curvature drifts-classically read at all orders in the expansion of the gyroaverage operator

$$
\begin{gathered}
\mathbf{v}_{\mathbf{E} \times \mathbf{B}}=\frac{1}{B_{\|}^{*}} \mathbf{b} \times \nabla \bar{\phi} \\
\mathbf{v}_{\nabla \boldsymbol{B}}=\frac{\mathbf{b}}{e B_{\|}^{*}} \times\left(\mu B \frac{\nabla B}{B}\right) \\
\mathbf{v}_{\boldsymbol{c}}=\frac{\mathbf{b}}{e B_{\|}^{*}} \times\left(m v_{\|}^{2} \frac{\mathrm{N}}{R}\right)
\end{gathered}
$$

where $\mathbf{N}$ is locally transverse to $\mathbf{B}$ the magnetic field, $\mathbf{N} / R=\nabla_{\perp} B / B+\nabla_{\perp} p /\left(B^{2} / \mu_{0}\right)$, and $v$ is the velocity along the field lines. Gyroaveraged quantities are represented with a bar and are either computed through a Padé approximation in GySELA ${ }^{38}$ or a four-point average technique in XGC1. ${ }^{52}$ The approximation of the integral over the gyroring by a sum over four points is rigorously equivalent to considering the Taylor expansion of the Bessel function at order two in the small argument limit, namely $J_{0}\left(k_{\perp} \rho_{s}\right) \approx 1-\left(k_{\perp} \rho_{s}\right)^{2} / 4$, and to computing the transverse Laplacian at second order using finite differences. ${ }^{53}$ 
${ }^{1}$ G. Dif-Pradalier, P. H. Diamond, V. Grandgirard, Y. Sarazin, J. Abiteboul, X. Garbet, Ph. Ghendrih, A. Strugarek, S. Ku, and C. S. Chang, Phys. Rev. E 82(2), 025401(R) (2010).

${ }^{2}$ G. Dif-Pradalier, V. Grandgirard, Y. Sarazin, X. Garbet, and Ph. Ghendrih, Phys. Rev. Lett. 103(6), 065002 (2009).

${ }^{3}$ X. Garbet, L. Laurent, A. Samain, and J. Chinardet, Nucl. Fusion 34(7), 963 (1994).

${ }^{4}$ P. H. Diamond and T. S. Hahm, Phys. Plasmas 2(10), 3640 (1995).

${ }^{5}$ B. A. Carreras, D. Newman, V. E. Lynch, and P. H. Diamond, Phys. Plasmas 3(8), 2903 (1996).

${ }^{6}$ X. Garbet and R. E. Waltz, Phys. Plasmas 5(8), 2836 (1998).

${ }^{7}$ P. A. Politzer, Phys. Rev. Lett. 84(6), 1192 (2000).

${ }^{8}$ P. Beyer, S. Benkadda, X. Garbet, and P. H. Diamond, Phys. Rev. Lett. 85(23), 4892 (2000).

${ }^{9}$ T. S. Hahm, P. H. Diamond, Z. Lin, G. Rewoldt, Ö. D. Gürcan, and S. Ethier, Phys. Plasmas 12(9), 090903 (2005).

${ }^{10}$ R. Sanchez, B. Ph. van Milligen, and B. A. Carreras, Phys. Plasmas 12(5), 056105 (2005).

${ }^{11}$ D. del Castillo-Negrete, Phys. Plasmas 13(8), 082308 (2006).

${ }^{12}$ I. Calvo, L. Garcia, B. A. Carreras, R. Sanchez, and B. Ph. van Milligen, Phys. Plasmas 15(4), 042302 (2008).

${ }^{13}$ K. W. Gentle, R. V. Bravenec, G. Cima, H. Gasquet, G. A. Hallock, P. E. Phillips, D. W. Ross, W. L. Rowan, A. J. Wootton, T. P. Crowley, J. Heard, A. Ouroua, P. M. Schoch, and C. Watts, Phys. Plasmas 2(6), 2292 (1995).

${ }^{14}$ P. Galli, G. Gorini, P. Mantica, G. M. D. Hogeweij, J. de Kloe, N. J. Lopes Cardozo, and RTP Team, Nucl. Fusion 39(10), 1355 (1999).

${ }^{15}$ P. Mantica, P. Galli, G. Gorini, G. M. D. Hogeweij, J. de Kloe, N. J. Lopes Cardozo, and RTP Team, Phys. Rev. Lett. 82(25), 5048 (1999).

${ }^{16}$ F. Ryter, R. Neu, R. Dux, H.-U. Fahrbach, F. Leuterer, G. Pereverzev, J. Schweinzer, J. Stober, W. Suttrop, ASDEX Upgrade Team, F. De Luca, A. Jacchia, and J. E. Kinsey, Nucl. Fusion 40(11), 1917 (2000).

${ }^{17}$ B. P. van Milligen, E. de la Luna, F. L. Tabares, E. Ascasibar, T. Estrada, F. Castejon, J. Castellano, I. Garcia-Cortes, J. Herranz, C. Hidalgo, J. A. Jimenez, F. Medina, M. Ochando, I. Pastor, M. A. Pedrosa, D. Tafalla, L. Garcia, R. Sanchez, A. Petrov, K. Sarksian, and N. Skvortsova, Nucl. Fusion 42(7), 787 (2002).

${ }^{18}$ C. C. Petty, T. C. Luce, K. H. Burrell, S. C. Chiu, J. S. deGrassie, C. B. Forest, P. Gohil, C. M. Greenfield, R. J. Groebner, R. W. Harvey, R. I. Pinsker, R. Prater, R. E. Waltz, R. A. James, and D. Wroblewski, Phys. Plasmas 2(6), 2342 (1995).

${ }^{19}$ T. C. Luce, C. C. Petty, and J. C. M. de Haas, Phys. Rev. Lett. 68(1), 52 (1992).

${ }^{20}$ C. C. Petty and T. C. Luce, Nucl. Fusion 34(1), 121 (1994).

${ }^{21}$ X. Garbet, G. Dif-Pradalier, C. Nguyen, Y. Sarazin, V. Grandgirard, and Ph. Ghendrih, Phys. Plasmas 16(6), 062503 (2009).

${ }^{22}$ F. L. Hinton and R. D. Hazeltine, Rev. Mod. Phys. 48(2), 239 (1976).

${ }^{23}$ T.-H. Watanabe and H. Sugama, Phys. Plasmas 11(4), 1476 (2004).

${ }^{24}$ A. A. Schekochihin, S. C. Cowley, W. Dorland, G. W. Hammett, G. G. Howes, E. Quataert, and T. Tatsuno, e-print arXiv:0704.0044v2.

${ }^{25}$ P. H. Diamond, S.-I. Itoh, K. Itoh, and T. S. Hahm, Plasma Phys. Controlled Fusion 47, R35 (2005).

${ }^{26}$ M. N. Rosenbluth and F. L. Hinton, Phys. Rev. Lett. 80, 724 (1998).

${ }^{27}$ F. L. Hinton and M. N. Rosenbluth, Plasma Phys. Controlled Fusion 41, A653 (1999).

${ }^{28}$ L. A. Charlton, B. A. Carreras, V. E. Lynch, K. L. Sidikman, and P. H. Diamond, Phys. Plasmas 1(8), 2700 (1994).

${ }^{29}$ Z. Lin, T. S. Hahm, W. W. Lee, W. M. Tang, and P. H. Diamond, Phys. Rev. Lett. 83(18), 3645 (1999).

${ }^{30}$ G. L. Falchetto and M. Ottaviani, Phys. Rev. Lett. 92(2), 025002 (2004).

${ }^{31}$ R. J. Taylor, M. L. Brown, B. D. Fried, H. Grote, J. R. Liberati, G. J. Morales, P. Pribyl, D. Darrow, and M. Ono, Phys. Rev. Lett. 63(21), 2365 (1989).

${ }^{32}$ K. H. Burrell, E. J. Doyle, P. Gohil, R. J. Groebner, J. Kim, R. J. La Haye, L. L. Lao, R. A. Moyer, T. H. Osborne, W. A. Peebles, C. L. Rettig, T. H. Rhodes, and D. M. Thomas, Phys. Plasmas 1, 1536 (1994).

${ }^{33}$ R. E. Bell, F. M. Levinton, S. H. Batha, E. J. Synakowski, and M. C. Zarnstorff, Phys. Rev. Lett. 81, 1429 (1998).

${ }^{34}$ K. Crombé, Y. Andrew, M. Brix, C. Giroud, S. Hacquin, N. C. Hawkes, A. Murari, M. F. F. Nave, J. Ongena, V. Parail, G. Van Oost, I. Voitsekhovitch, and K.-D. Zastrow, Phys. Rev. Lett. 95, 155003 (2005).

${ }^{35}$ R. M. McDermott, B. Lipschultz, J. W. Hughes, P. J. Catto, A. E. Hubbard, I. H. Hutchinson, R. S. Granetz, M. Greenwald, B. LaBombard, K.
Marr, M. L. Reinke, J. E. Rice, D. Whyte, and Alcator C-Mod Team, Phys. Plasmas 16(5), 056103 (2009).

${ }^{36}$ P. H. Diamond, Y. M. Liang, B. A. Carreras, and P. W. Terry, Phys. Rev. Lett. 72(16), 2565 (1994).

${ }^{37}$ C. J. McDevitt, P. H. Diamond, O. D. Gurcan, and T. S. Hahm, Phys. Plasmas 17(11), 112509 (2010).

${ }^{38}$ V. Grandgirard, M. Brunetti, P. Bertrand, N. Besse, X. Garbet, Ph. Ghendrih, G. Manfredi, Y. Sarazin, O. Sauter, E. Sonnendrücker, J. Vaclavik, and L. Villard, J. Comput. Phys. 217(2), 395 (2006).

${ }^{39}$ J. A. Heikkinen, S. J. Janhunen, T. P. Kiviniemi, and F. Ogando, J. Comput. Phys. 227(11), 5582 (2008).

${ }^{40}$ S. Ku, C. S. Chang, M. Adams, J. Cummings, F. Hinton, D. Keyes, S. Klasky, W. Lee, Z. Lin, S. Parker, and the CPES team, J. Phys.: Conf. Ser. 46, 87 (2006).

${ }^{41}$ B. F. McMillan, S. Jolliet, T. M. Tran, L. Villard, A. Bottino, and P. Angelino, Phys. Plasmas 15(5), 052308 (2008).

${ }^{42}$ B. D. Scott, in Proceedings of the EPS Conference, Hersonissos, Greece, 2008.

${ }^{43}$ X. Q. Xu, Z. Xiong, Z. Gao, W. M. Nevins, and G. R. McKee, Phys. Rev. Lett. 100(21), 215001 (2008).

${ }^{44}$ Y. Idomura, H. Urano, N. Aiba, and S. Tokuda, Nucl. Fusion 49(6), 065029 (2009).

${ }^{45}$ S. Ku, C. S. Chang, and P. H. Diamond, Nucl. Fusion 49(11), 115021 (2009).

${ }^{46}$ Y. Idomura, S. Tokuda, and Y. Kishimoto, Nucl. Fusion 43(4), 234 (2003).

${ }^{47}$ P. Angelino, A. Bottino, R. Hatzky, S. Jolliet, O. Sauter, T. M. Tran, and L. Villard, Phys. Plasmas 13(5), 052304 (2006).

${ }^{48}$ G. Dif-Pradalier, V. Grandgirard, Y. Sarazin, X. Garbet, Ph. Ghendrih, and P. Angelino, Phys. Plasmas 15, 042315 (2008).

${ }^{49}$ G. Dif-Pradalier, P. H. Diamond, V. Grandgirard, Y. Sarazin, J. Abiteboul, X. Garbet, Ph. Ghendrih, G. Latu, A. Strugarek, S. Ku, and C. S. Chang, "Modeling choices in numerical simulations: where do we stand in terms of the physics?" (submitted).

${ }^{50}$ C. S. Chang, S. Ku, P. H. Diamond, Z. Lin, S. Parker, T. S. Hahm, and N. Samatova, Phys. Plasmas 16(5), 056108 (2009).

${ }^{51}$ Y. Sarazin, V. Grandgirard, J. Abiteboul, S. Allfrey, X. Garbet, Ph. Ghendrih, G. Latu, A. Strugarek, and G. Dif-Pradalier, Nucl. Fusion 50(5), 054004 (2010).

${ }^{52}$ Z. Lin and W. W. Lee, Phys. Rev. E 52(5), 5646 (1995).

${ }^{53} \mathrm{~V}$. Grandgirard and Y. Sarazin, "Gyrokinetic simulations of magnetic fusion plasmas," (submitted).

${ }^{54}$ Y. Sarazin and Ph. Ghendrih, Phys. Plasmas 5(12), 4214 (1998).

${ }^{55}$ X. Garbet, Y. Sarazin, P. Beyer, Ph. Ghendrih, R. E. Waltz, M. Ottaviani, and S. Benkadda, Nucl. Fusion 39, 2063 (1999).

${ }^{56}$ J. Abiteboul, X. Garbet, V. Grandgirard, S. J. Allfrey, G. Dif-Pradalier, Ph. Ghendrih, G. Latu, C. Passeron, Y. Sarazin, and A. Strugarek, in Proceedings of the 37th EPS conference on plasma physics, Dublin, 2010.

${ }^{57}$ G. Latu, N. Crouseilles, V. Grandgirard, and E. Sonnendrücker, "Gyrokinetic semi-Lagrangian parallel simulation using a hybrid openMP/MPI programming," in Recent Advances in Parallel Virtual Machine and Message Passing Interface, European PVM/MPI Users' Group Meeting, edited by F. Capello, T. Herault, and J. Dongarra (Springer, BerlinHeidelberg, 2007), Vol. 14, pp. 356-364.

${ }^{58}$ N. Crouseilles, G. Latu, and E. Sonnendrücker, J. Comput. Phys. 228, 1429 (2009).

${ }^{59}$ W. X. Wang, N. Nakajima, M. Okamoto, and S. Murakami, Plasma Phys. Controlled Fusion 41(9), 1091 (1999).

${ }^{60}$ X. Q. Xu and M. N. Rosenbluth, Phys. Fluids B 3(3), 627 (1991)

${ }^{61}$ A. M. Dimits and B. I. Cohen, Phys. Rev. E 49(1), 709 (1994).

${ }^{62}$ Z. Lin, W. M. Tang, and W. W. Lee, Phys. Plasmas 2(8), 2975 (1995).

${ }^{63}$ A. H. Boozer and G. Kuo-Petravic, Phys.Fluids 24(5), 851 (1981).

${ }^{64}$ C. S. Chang, S. Ku, and H. Weitzner, Phys. Plasmas 11, 2649 (2004).

${ }^{65}$ C. S. Chang and S. Ku, Contrib. Plasma Phys. 46, 496 (2006).

${ }^{66}$ C. S. Chang and S. Ku, Phys. Plasmas 15(6), 062510 (2008).

${ }^{67}$ Y. B. Kim, P. H. Diamond, H. Biglari, and J. D. Callen, Phys. Fluids B, 3(2), 384 (1991).

${ }^{68}$ Y. B. Kim, P. H. Diamond, and R. J. Groebner, Phys. Fluids B 3(8), 2050 (1991).

${ }^{69}$ F. I. Parra and P. J. Catto, Plasma Phys. Controlled Fusion 50(6), 065014 (2008).

${ }^{70}$ M. Yoshida, Y. Koide, H. Takenaga, H. Urano, N. Oyama, K. Kamiya, Y. Sakamoto, Y. Kamada, and the JT-60 Team, Plasma Phys. Controlled Fusion 48(11), 1673 (2006). 
${ }^{71}$ E. Trier, L.-G. Eriksson, P. Hennequin, C. Fenzi, C. Bourdelle, G. Falchetto, X. Garbet, T. Aniel, F. Clairet, and R. Sabot, Nucl. Fusion 48(9), 092001 (2008).

${ }^{72}$ Ö. D. Gürcan, P. H. Diamond, T. S. Hahm, and R. Singh, Phys. Plasmas 14(4), 042306 (2007).

${ }^{73}$ C. Hidalgo, B. Gonçalves, C. Silva, M. A. Pedrosa, K. Erents, M. Hron, and G. F. Matthews, Phys. Rev. Lett. 91(6), 065001 (2003).

${ }^{74}$ B. Gonçalves, C. Hidalgo, M. A. Pedrosa, R. O. Orozco, E. Sánchez, and C. Silva, Phys. Rev. Lett. 96(14), 145001 (2006).

${ }^{75}$ P. H. Diamond, C. J. McDevitt, Ö. D. Gürcan, T. S. Hahm, W. X. Wang, E. S. Yoon, I. Holod, Z. Lin, V. Naulin, and R. Singh, Nucl. Fusion 49(4), 045002 (2009).

${ }^{76}$ A. Bortolon, B. P. Duval, A. Pochelon, and A. Scarabosio, Phys. Rev. Lett. 97(23), 235003 (2006)

${ }^{77}$ W. M. Solomon, K. H. Burrell, J. S. deGrassie, R. Budny, R. J. Groebner, J. E. Kinsey, G. J. Kramer, T. C. Luce, M. A. Makowski, D. Mikkelsen, R. Nazikian, C. C. Petty, P. A. Politzer, S. D. Scott, M. A. Van Zeeland, and M. C. Zarnstorff, Plasma Phys. Controlled Fusion 49(12B), B313 (2007).

${ }^{78}$ K. Ida, M. Yoshinuma, K. Nagaoka, M. Osakabe, S. Morita, M. Goto, M. Yokoyama, H. Funaba, S. Murakami, K. Ikeda, H. Nakano, K. Tsumori,
Y. Takeiri, O. Kaneko, and LHD experiment group, Nucl. Fusion 50(6), 064007 (2010).

${ }^{79}$ S. Ku, G. Dif-Pradalier, S. M. Yi, E. S. Yoon, P. H. Diamond, T. S. Hahm, W. Solomon, C. S. Chang, Y. Sarazin, V. Grandgirard, J. Abiteboul, X. Garbet, Ph. Ghendrih, G. Latu, and A. Strugarek, in Proceedings of the 23rd IAEA Fusion Energy Conference, 2010.

${ }^{80}$ J. Abiteboul, X. Garbet, V. Grandgirard, S. Allfrey, Ph. Ghendrih, G. Latu, Y. Sarazin, and A. Strugarek, "Conservation equations and calculation of mean flows in gyrokinetics," Phys. Plasmas (submitted).

${ }^{81}$ E. A. Belli and J. Candy, Plasma Phys. Controlled Fusion 50(9), 095010 (2008).

${ }^{82}$ F. L. Hinton and Y.-B. Kim, Phys. Plasmas 2(1), 159 (1995).

${ }^{83}$ M. Taguchi, Plasma Phys. Controlled Fusion 30(13), 1897 (1988).

${ }^{84}$ S. P. Hirshman and D. J. Sigmar, Phys. Fluids 19(10), 1532 (1976).

${ }^{85}$ C. S. Chang and F. L. Hinton, Phys. Fluids 25(9), 1493 (1982).

${ }^{86}$ C. S. Chang and F. L. Hinton, Phys. Fluids 29(10), 3314 (1986).

${ }^{87} \mathrm{~S}$. Chapman and T. G. Cowling, The Mathematical Theory of NonUniform Gases (University Press, New York, 1952).

${ }^{88}$ H. Sugama and T.-H. Watanabe, Phys. Plasmas 13(1), 012501 (2006). 